\title{
Antimicrobial Resistance and Molecular Patterns in Community-acquired Complicated Intra-abdominal Infections: A Multicentric Study
}

\author{
Vildan Avkan-Oğuz (iD, Nurcan Baykam² (D), Volkan Korten³ (D), Madina Abdullayeva ${ }^{\mathbf{1}}$ (D), Derya Yapar² (D), \\ Lütfıye Mülazımoğlu ${ }^{3}$, Zeynep Gülay ${ }^{4}$ \\ 1 Department of Infectious Diseases and Clinical Microbiology, Dokuz Eylül University School of Medicine, İzmir, Turkey \\ 2 Department of Infectious Diseases and Clinical Microbiology, Hitit University School of Medicine, Çorum, Turkey \\ 3 Department of Infectious Diseases and Clinical Microbiology, Marmarat University School of Medicine, İstanbul, Turkey \\ 4 Department of Medical Microbiology, Dokuz Eylül University School of Medicine, İzmir, Turkey
}

\begin{abstract}
Objective: We aimed to analyze antimicrobial susceptibilities by a molecular evaluation of extended-spectrum beta-lactamase (ESBL) positive of the isolates from communityacquired complicated intra-abdominal infections (CA- IAIs) in Turkey.

Method: Clinical samples were obtained during operation. Antimicrobial susceptibilities, inducible beta-lactamase and ESBL status, were determined using Clinical and Laboratory Standards Institute criteria and interpretive standards. ESBL positive and cefoxitin-resistant isolates were evaluated bla genes for CTX-M, TEM, SHV, PER-1 and plasmidic AmpC families with polymerase chain reaction (PCR). We confirmed the results by directly sequencing the bla genes (Macrogen Inc, Korea) with Mega 5.02 and BLAST programs.

Results: We isolated 116 pathogens from 81 patients. Clinicians diagnosed 34 (42.1\%) patients as acute appendicitis, $15(18.5 \%)$ as cholecystitis, 14 (17.3\%) as intra-abdominal abscess, 12 (14.8\%) as tumor resection and six (7.3\%) acute diverticulitis. Escherichia coli (E. coli) was the most common gram-negative (76\%), Enterococcus spp. was the most common gram-positive (13.6\%). ESBL production was 12, $3 \%$ in all gram-negative strains; $11,1 \%$ (9/62) in E. coli and 1, 2\% (1/9) Klebsiella pneumoniae (K. pneumonia). Quinolone resistance was $22.2 \%$ and ceftriaxone resistance was $14.5 \%$ in E. coli. We detected CTX-M genes in five of nine ESBL positive isolates. CTX-M-1 group (CTX-M-1, CTX-M-3, and CTX-M-15) was in four and CTX-M-9 group (CTX-M-14) in one ESBL positive E. coli. One isolate had also AmpC, CMY-2 enzyme (1, 6 \%).

Conclusion: In our study, ESBL positive gram-negative pathogens were $>10 \%$. Quinolone resistance was $>\% 20$, so that quinolones should not be the first choice for the treatment of serious IAI's in our country. Cefoxitin resistance was still low in E. coli isolates from CA-IAIs. Regular surveillance data can guide empirical antibiotic therapy in community-acquired intra-abdominal infections. It should emphasize the importance of sampling for culture to surgeons for guiding empirical therapy in the future.
\end{abstract}

Keywords: Community-acquired complicated intra-abdominal infections, ExtendedSpectrum Beta-Lactamase, cefoxitin resistance, CTX-M
Corresponding Author:

Vildan Avkan-Oğuz

E-mail:

vildan.oguz@gmail.com

Received: July 22, 2020

Accepted: August 21, 2020

Published: August 31, 2020

\section{Suggested citation:}

Avkan-Oğuz V, Baykam N, Korten V, Abdullayeva M, Yapar D,

Mulazımoğlu L et al. Antimicrobial Resistance and Molecular

Patterns in Community-acquired Complicated Intra-abdominal Infections: A Multicentric Study. Infect Dis Clin Microbiol 2020; 2: 71-77.

DOI: $10.36519 / \mathrm{idcm} .2020 .0018$ 


\section{INTRODUCTION}

$\square$ omplicated intra-abdominal infection (CIAI) is defined as localized or diffuse peritonitis with the involvement of multiple intraperitoneal organs $(1,2)$. Source control and appropriate empirical antibiotics are essentials of the treatment. There are few clinical and microbiologic studies regarding cIAI in Turkey $(3,4)$. Empiric antibiotics were selected due to diagnoses; community-acquired IAI (CA-IAI) or healthcare-associated IAI. Guidelines published on this topic lack definitive criteria for CA-IAI $(1,2)$. Previous interventions and hospitalization must be ascertained. In light of this information, we defined CA-IAI based on multiple criteria and conducted this study to contribute to local microbiological and antimicrobial susceptibility data in Turkey.

\section{METHODS}

We defined CA-IAI, as patients had no history of hospital stay and abdominal procedure in the last three months. Eighty-one adult patients were included in the study from three tertiary hospitals in Marmara (39, \%49), Aegean (34, \%42), and the central Anatolia region $(8, \% 9.9)$. The total bed capacities of the hospitals vary from 640 to 1005, with 42-63 beds in the general surgery department and about 50 beds on average in the adult intensive care units. In each center, only samples obtained by intraoperative or percutaneous drainage were evaluated. The patients' age, gender, primary disease, comorbidities, type of surgical intervention (operation or drainage), length of hospital stay, and culture and antimicrobial susceptibility results were recorded. Superficial specimens and isolated from in situ drains or drainage bottles were excluded. Although Marmara (3) and Aegean study centers (6) had extended-spectrum beta-lactamase-producing (ESBL-producing) strains, the central Anatolia center had no resistant strain. ESBL-producing strains and cefoxitin-resistant strains were collected at a single center for molecular analysis.

Specimen culture, identification, and antimicrobial susceptibility: Bacterial identification was performed by routine phenotypic methods when growth was detected in cultures of abscess mate- rial and peritoneal fluid samples obtained from the infected area by an intraoperative and percutaneous drain. Antimicrobial susceptibilities were evaluated according to Clinical and Laboratory Standards Institute (CLSI) recommendations. Inducible beta-lactamase (IBL) and ESBL production were evaluated using the combined disk method and automated system panels (5). Intrinsically susceptible isolates, which were phenotypically positive for screened resistance patterns, were preserved at the individual centers and sent to the central laboratory for molecular investigation of the beta-lactamases.

Molecular characterization of beta-lactamase genes: Presence of bla genes for CTX-M group (CTX-M-1, -2, -8, -9), TEM, SHV, and PER-1 and plasmidic AmpC families (MOX, CIT, DHA, ACC, EBC, and FOX) was evaluated in ESBL-producing and cefoxitin-resistant isolates, respectively, using PCR methodology as described previously (6-8). The results were confirmed by direct sequencing of both strands of the bla genes (Macrogen Inc, Korea) and analyzing the resulting sequences with Mega 5.02 and BLAST programs.

\section{RESULTS}

We evaluated 81 patients with positive cultures. The mean age was 53.94 \pm 19.49 years (19-88), and 46 $(56.8 \%)$ of the patients were male. Clinicians diagnosed 34 (42.1\%) patients as acute appendicitis, 15

\section{HIGHLIGHTS}

- This study is the first to evaluate the clinical features and molecular resistance patterns in community-acquired intra-abdominal infections in Turkey.

- ESBL-production (especially CTX-M-14 and CTX-M-15) was over 10\% of Enterobacteriaceae.

- In Escherichia coli isolates from complicated Intra-abdominal Infections, resistance to quinolone and ceftriaxone were $22.2 \%$ and $14.5 \%$, respectively.

- Sampling for culture during surgery is helpful in guiding empirical antimicrobial therapy. 
Table 1. The distribution of microorganism according to the infection site

\begin{tabular}{|c|c|c|c|c|c|c|}
\hline Microorganisms & Appendicular & $\begin{array}{l}\text { Tumour } \\
\text { resection }\end{array}$ & Abscess & Cholecystitis & Diverticulitis & $\begin{array}{c}\text { Total } \\
n=116\end{array}$ \\
\hline E. coli / ESBL + & $32 / 3$ & $7 / 1$ & $7 / 3$ & $7 / 1$ & $-/ 1$ & $53 / 9$ \\
\hline P. aeruginosa / IBL + & $2 / 4$ & $2 / 1$ & $0 / 3$ & - & $0 / 1$ & $4 / 9$ \\
\hline K. pneumonia / ESBL + & $1 /-$ & - & $2 / 1$ & $2 /-$ & $2 /-$ & $7 / 1$ \\
\hline K. oxytoca & - & - & - & 1 & - & 1 \\
\hline E. aerogenes & & & & 1 & & 1 \\
\hline E. cloacea $\quad \mathrm{IBL}+$ & & & & 3 & 2 & 5 \\
\hline Citrobacter spp IBL+ & & 1 & & & 1 & 2 \\
\hline M. morgani $\quad$ IBL+ & 1 & & & 1 & & 2 \\
\hline Enterococus spp & 2 & 3 & 1 & 5 & & 11 \\
\hline Streptococcus spp & 5 & & & 1 & & 6 \\
\hline MRSA & & 1 & & & & 1 \\
\hline B. fragilis & 1 & 2 & & & 1 & 4 \\
\hline
\end{tabular}

Table 2. Antibiotic susceptibility rates (\%) for Gram negative isolates in community acquired intra-abdominal infections

\begin{tabular}{|l|c|c|c|c|c|c|c|c|c|c|}
\hline & $\mathbf{n}$ & $\begin{array}{c}\text { ESBL } \\
(\%)\end{array}$ & AM & $\begin{array}{c}\text { TMP- } \\
\text { SXT }\end{array}$ & CIP & AMC & CRO & TZP & CAZ & AMK \\
\hline E. coli & 62 & 11,1 & 41,9 & 56,5 & 77,4 & 80.0 & 85.5 & 88,7 & - & 93,5 \\
\hline Klebsiella spp. & 9 & 1,2 & 0.0 & 100 & 88,9 & 88,9 & 100 & 88,9 & - & 100 \\
\hline P. aeruginosa & 13 & - & - & - & 84,9 & - & - & 92,3 & 100 & 100 \\
\hline
\end{tabular}

ESBL: Extended spectrum beta lactamases; AM: ampicillin; TMP-SXT: trimethoprim-sulfamethoxazole; CIP: ciprofloxcasin; AMC: amoxicillin/clavulanic acid; CRO: cefriaxone; TZP: piperacilin/tazobactam, CAZ: ceftazidime; AMK: amikasin

$(18.5 \%)$ as cholecystitis, $14(17.3 \%)$ as intra-abdominal abscess, $12(14.8 \%)$ as tumor resection and six $(7.3 \%)$ acute diverticulitis. Twelve $(14.8 \%)$ patients had malignancy, and 10 (12.3\%) had diabetes mellitus. None of the patients had used antibiotics in the past month. The mean length of hospital stay was $8.38 \pm 6.15$ days (1-30 days).

We isolated 116 pathogens belonging to 12 different species. The most common gram-negative pathogen was Escherichia coli (E. coli) 62 (76.5\%), gram-positive pathogens were Enterococcus spp. 11 (13.6\%). The distribution of microorganisms, according to the infection site, was shown in Table 1. ESBL production was detected in $10(12.3 \%)$ isolates, nine $(11.1 \%)$ in E. coli and one (1.2\%) in Klebsiella spp. IBL production was detected in 18 (22.2\%) isolates (in Pseudomonas aeruginosa, Citrobacter spp., Morganella morganii, Enterobacter spp.). The rate of ESBL positivity in all E. coli strains was $14.5 \%$ (9/62). Of the nine ESBL-producing E. coli, 7 (77.7\%) were resistant to ceftriaxone, 5 (55.5\%) to trimethoprim-sulfamethoxazole, and $3(33.3 \%)$ to quinolone. Mixed infection was in twenty-seven cultures (33.3\%). There was no ESBL or IBL production in isolates from the center in the central Anatolia region. Antimicrobial 
Table 3. The features of patients with ESBL positive E. coli

\begin{tabular}{|c|c|c|c|c|c|c|c|}
\hline Patient & Age & Sex & $\begin{array}{l}\text { Type of } \\
\text { infection }\end{array}$ & Type of ESBL & Amp C & Ceftriaxone & Quinolone \\
\hline 1 & 28 & $\mathrm{~F}$ & Diverticulitis & TEM 1 & Negative & Sensitive & Sensitive \\
\hline 2 & 53 & $\mathrm{~F}$ & Tumor resection & $\begin{array}{c}\text { TEM1 } \\
\text { CTX-M } 15\end{array}$ & Positive (CMY 2) & Resistant & Resistant \\
\hline 3 & 44 & $\mathrm{~F}$ & Appendicular & TEM 1 & Negative & Resistant & Sensitive \\
\hline 4 & 21 & $\mathrm{~F}$ & Appendicular & TEM 2 & Negative & Sensitive & Sensitive \\
\hline 5 & 33 & $\mathrm{~F}$ & Abscess & $\begin{array}{c}\text { TEM } 1 \\
\text { CTX-M } 14 \\
\text { CTX-M } 15\end{array}$ & Positive & Resistant & Resistant \\
\hline 6 & 68 & $\mathrm{~F}$ & Cholecystitis & $\begin{array}{l}\text { TEM } 1 \\
\text { TEM } 2\end{array}$ & Negative & Resistant & Sensitive \\
\hline 7 & 66 & $\mathrm{~F}$ & Appendicular & $\begin{array}{c}\text { TEM1 } \\
\text { CTX-M } 1\end{array}$ & Negative & Resistant & Resistant \\
\hline 8 & 39 & M & Abscess & $\begin{array}{c}\text { TEM1 } \\
\text { CTX-M } 3\end{array}$ & Negative & Sensitive & Resistant \\
\hline 9 & 52 & $\mathrm{~F}$ & Abscess & $\begin{array}{l}\text { TEM1 } \\
\text { CTX-M } 3 \\
\text { SHV } 1\end{array}$ & Negative & Resistant & Sensitive \\
\hline
\end{tabular}

ESBL: Extended spectrum beta lactamases

susceptibility patterns of the gram-negative isolates were shown in Table 2.

The molecular analysis for the beta-lactamases revealed that all ESBL-positive E. coli isolates produced one or both of TEM-1 and TEM-2 broad-spectrum beta-lactamases. Five (55.5\%) isolates produced CTX-M-type ESBLs (CTX-M-1 group [CTX-M-1, CTX-M-3, and CTX-M-15] in four and CTX-M-9 group [CTX-M-14] in one). Mutant PER-1 enzyme genes were not detected in any of the isolates.

Two of the E. coli isolates were both ESBL-producing and cefoxitin-resistant. In two cefoxitin-resistant isolates, pampC gene analysis was performed by multiplex polymerase chain reaction (PCR), and CMY-2 enzyme was detected in one $(1,6 \%)$ isolate. This strain was isolated from a 53-year-old female patient admitted to the emergency department due to abdominal pain, nausea, and hypotension. On computerized abdominal tomography, an extraluminal mass lesion reaching about $10 \mathrm{~cm}$ in diam- eter and invading the iliopsoas muscle was detected in the descending colon. Perforation associated with sigmoid tumor invasion of the psoas muscle was observed, and the patient underwent surgery. Pathologic diagnosis was mucinous adenocarcinoma (>50\% mucinous). Perioperative peritoneal culture of material obtained during surgery yielded ESBL-producing E. coli. Multiple beta-lactamase genes (TEM 1, CTXM-1, CTX-M 15, and CMY-2) were detected in this agent. The characteristics of the patients with ESBL positivity and cefoxitin resistance are presented in Table 3.

\section{DISCUSSION}

This study is the first conducted in our country to present clinical findings in CA-IAI patients together with the causative agents and their resistance features. In our study, E. coli was the most common (76\%) gram-negative agent. ESBL production was $11.1 \%$ of all strains isolated, $14.5 \%$ of E. coli strains. In perforated appendicitis in children, ESBL rate 
was reported $56.7 \%$ and $31.2 \%$ in E.coli $(9,10)$. In SMART study in Turkey, researchers did not present CA IAIs; however, ESBL rates in intensive care unit IAIs were $29.2 \%$ (3). In an international study including Turkey, in which 1645 patients with community-acquired IAIs were evaluated, ESBL rate was 12, 2\% (56/456) in E. coli isolates (11). We suggest that ESBL production in CA-IAIs can be a threat to our country.

Five of nine (55.5\%) ESBL-producing E. coli had CTX-M genes; two (22.2\%) isolates had CTX-M-14 and CTX-15. In China, although community or hospital-acquired IAIs were undefined, writers reported CTX-M 15 was $62.3 \%$ in E. coli isolated from IAIs. (12). In SMART study in Asia, ESBL production was more than $30 \%$ in E. coli and K. pneumonia isolated from CA-IAIs; CTXM-14 and CTX-15 were the most prominent variant (13). CTXM-14 and CTXM-15 rates found in our study were lower than the rates of those studies.

We detected AmpC b-lactamase, CMY-2 enzyme in one $(1,6 \%)$ E. coli isolate (Table 3). It was quite low when we compared 38\% of bla CMY-2 in Enterobacteriaceae isolates from CA- IAI in Asia study (13). CMY-2 enzyme was reported before in different samples, for example, blood samples in one of our study centers (14). We demonstrated CTX-M genes and bla CMY-2 in E. coli strains from CA-IAI for the first time in Turkey. CMY-2-like enzymes are among the most common pAmpC enzymes worldwide and are reported to be precursors of other CMY variants $(15,16)$. pAmpCs can spread between species by horizontal migration. In our study, cefoxitin resistance was still low; it can be supported with further studies.

$22.6 \%$ of all E. coli strains and $33.3 \%$ of isolated ESBLpositive E. coli strains had quinolone resistance. Quinolone resistance is usually chromosomal, and it can also be spread via resistance genes (qnr) transferred by plasmids. The presence of transferrable quinolone resistance genes in Turkey has been investigated in various studies $(17,18)$. Although qnr genes were not investigated in the present study, these data suggest that clinicians should use quinolones cautiously in CA-IAIs.

Surgical infection society guideline suggests having samples for culture to tract epidemiologic changes in CA-IAIs. (19). In Turkey, perioperative culture rates in CIAI cases vary across operations. In a recent study in our country, surgeons took samples for culture in $94.7 \%$ of intra-abdominal abscess and $9.6 \%$ of acute appendicitis (20). In another study, pediatric surgeons had taken intraoperative cultures in $20 \%$ in acute and $67 \%$ perforated appendicitis (9). In an international study, intraperitoneal specimens were taken $59,4 \%$ in CA-IAIs (11). Infectious disease and clinical microbiology specialists should emphasize the importance of sampling for culture to guide empirical therapy in the future.

We had some limitations in our study, the most important of which is the small number of patients. Another shortcoming of the study was that to reduce costs, molecular analyses were not done for all strains, but only for resistant E. coli strains. We defined five enzymes molecularly of nine ESBL positive isolates. This can be a result of ESBL plasmid loss during stocking, the presence of a minor ESBL not included in molecular tests, or misinterpretation of the phenotypic test. Different studies reported similar situations $(21,22)$. Our data represent just three geographic regions, so we cannot generalize to the whole country.

In conclusion, we report more than 10\% ESBL rate in the gram-negatives isolated from CA-IAIs. Quinolone resistance was $>\% 20$, so that quinolones should not be the first choice for the treatment of serious IAI's in our country. We also suggest that ESBL production in CA-IAIs can be a threat in our country. Cefoxitin resistance rate was still low in $E$. coli isolates. To generate a countrywide epidemiologic data, surgeons should be aware of importance of sampling in CA-IAIs. This data can guide appropriate empiric antimicrobial therapy in the future. 
Peer-review: Externally peer-reviewed

Ethical Approval: Dokuz Eylul University Ethical Committee approved this noninvasive research with the decision number of 2018/28-26.

Author Contributions: Concept - V.A.O., N.B., V.K.; Design - V.A.O., Z.G., N.B., V.K.; Supervision - V.A.O., N.B., V.K.; Fundings - V.A.O., Z.G.; Materials - V.A.O., M.A., D.Y; Data Collection and/or Processing M.A., D.Y., V.A.O., ; Analysis and/or Interpretation - V.A.O., N.B., V.K.;
Literature Review - V.A.O., N.B., V.K., L.M.; Writer - V.A.O., N.B., V.K., L.M., Z.G.; Critical Reviews - V.A.O., V.K., N.B.

Conflict of Interest: The authors have no conflict of interest to declare.

Financial Disclosure: The authors declared that this study has received no financial support.

Acknowledgements: We thank all surgeons who took samples for our study.

\section{REFERENCES}

1 Solomkin JS, Mazuski JE, Bradley JS, Rodvold KA, Goldstein EJ, Baron EJ, et al. Diagnosis and management of complicated intra- abdominal infections in adults and children: guidlines by the Surgical Infection Society and the Infectious Diseases Society of America. Clin Infect Dis 2010; 50: 133-64.

2 Sartelli M, Viale P, Catena F, Ansaloni L, Moore E, Malangoni M, et al. 2013 WSES guidelines for management of intra-abdominal infections. World J Emerg Surg 2013; 8: 3.

3 Koksal I, Yilmaz G, Unal S, Zarakolu P, Korten V, Mulazimog lu L, et al. Epidemiology and susceptibility of pathogens from SMART 2011-12 Turkey: evaluation of hospital-acquired versus mmunity-acquired urinary tract infections and ICU-versus non-ICU-associated intra-abdominal infections. J Antimicrob Chemother 2017;72: 1364-72.

4 Avkan-Oguz V, Yapar N, Alp-Cavus S, Demir Onder K, Aktas E, Gulay Z, et al. Clinical and microbiological efficacy of tigecycline for complicated skin-soft-tissue and intra-abdominal infections in a Turkish university hospital. Int J Clin Pract 2013; 67: 505-11.

5 European Committee on Antimicrobial Susceptibility Testing. Breakpoint tables for interpretation of MICs and zone diameters. Version 7.0, 2017 (cited 2020 June 22). Available from: URL: http://eucast.org

6 Jeong SH, Bae IK, Kwon SB, Lee JH, Song JS, Jung HI, et al. Dissemination of transferable CTX-M-type extended-spectrum $\beta$-lactamase-producing Escherichia coli in Korea. J Appl Microbiol 2005: 98; 921-7.

7 Rodríguez-Baño J, Navarro MD, Romero L, Martínez-Martínez L , Muniain MA, Perea EJ, et al. Epidemiology and clinical features of infections caused by extended-spectrum beta-lactamase-producing Escherichia coli in nonhospitalized patients. J Clin Microbiol 2004; 42: 1089-94.

8 Perez-Perez FJ, Hanson ND. Detection of plasmid-mediated AmpC beta-lactamase genes in clinical isolates by using multiplex PCR. J Clin Microbiol 2002; 40(6): 2153-62.

9 Turel O, Mirapoglu SL, Yuksel M, Ceylan A, Gultepe BS. Perfo- rated appendicitis in children: Antimicrobial susceptibility and antimicrobial stewardship. J Glob Antimicrob Resist 2019; 16: 159-61.

10 Sayiner H, Akgün S, Apaydin HÖ, Göksu M, Aksoy N, Akgun I, et al. The importance of culture-antibiogram and evaluation of the empirical antibiotic treatment in peritonitis due to perforated appendicitis acquired intra-abdominal infection. Biomed Res 2018; 29: 1420-4.

11 Sartelli M, Catena F, Ansaloni L, Coccolini F, Corbella D, Moore $\mathrm{EE}$, et al. Complicated intra-abdominal infections worldwide: the definitive data of the CIAOW Study. World J Emerg Surg 2014; 9: 37 .

12 Liao K, Chen Y, Wang M, Guo P, Yang Q, Ni Yuxing, et al. Molecular characteristics of extended-spectrum $\beta$-lactamase-producing Escherichia coli and Klebsiella pneumoniae causing intra-abdominal infections from 9 tertiary hospitals in China. Diagn Microbiol Infect Dis 2017; 87: 45-8.

13 Jean SS, Hsueh PR; SMART Asia-Pacific Group. Distribution of ESBLs, AmpC $\beta$-lactamases and carbapenemases among Enterobacteriaceae isolates causing intra-abdominal and urinary tract infections in the Asia-Pacific region during 2008-14: results from the Study for Monitoring Antimicrobial Resistance Trends (SMART). J Antimicrob Chemother. 2017; 72:166-71.

14 Sarı AN, Biçmen M, Gülay Z. Escherichia coli ve Klebsiella pneumoniae kan kültürü izolatlarında plazmid aracılı AmpC beta-laktamaz varlığının araştırılması [Investigation of plasmid mediated AmpC beta-lactamases among Escherichia coli and Klebsiella pneumoniae isolated from blood cultures]. Mikrobiyol Bul 2013; 47: 582-91.

15 Jacoby GA. AmpC beta-lactamases. Clin Microbiol Rev 2009; 22: $161-82$.

16 Rodríguez-Baño J, Mingorance J, Fernández-Romero N, Serrano L, López-Cerero L, Pascual A, et al. Outcome of bacteraemia due to extended-spectrum $\beta$-lactamase-producing Escherichia coli: Impact of microbiological determinants. J Infect 2013; 67: $27-34$. 
17 Coban AY, Nohut OK, Tanrıverdi Çaycı Y, Bayramoğlu G, Pirinççiler M, Cetinkaya E, et al. Enterobacteriaceae üyelerinde plasmid aracılı kinolon direnç determinantlarının araştırılması: çok merkezli bir çalışma [Investigation of quinolone resistance determinants in Enterobacteriaceae: a multicentre study]. Mikrobiol Bul 2012; 46: 366-74.

18 Hoşgör-Limoncu M, Eraç B, Yurtman A, Aydemir S. Plasmid-mediated quinolone resistance mechanismsin ESBL pos itive Escherichia coli and Klebsiella pneumoniae strains at a tertiary-care hospital in Turkey. J Chemother 2012; 24:144-9.

19 Mazuski JE, Tessier JM, May AK, Sawyer RG, Nadler EP, Rosengart MR, et al. The surgical infection society revised guidelines on the management of intra-abdominal infection. Surg Infect 2017; 18: 1-76.

20 Avkan-Oğuz V, Baykam N, Sökmen S, Güner R, Agalar F, Alp E, et al. Recommendations for intra-abdominal infections consensus report. Ulus Cerrahi Derg 2016 ;32:306-21.

21 Yavuz B, Ozer B, Inci M, Duran N. Determination of CTX-M beta-lactamase in Escherichia coli strains isolated from clinical samples. Infez Med 2015; 23: 23-30.

22 Copur Cicek A, Saral A, Ozad Duzgun A, Yasar E, Cizmeci Z, Ozlem Balci P, et al. Nationwide study of Escherichia coli producing extended-spectrum $\beta$-lactameses TEM, SHV and CTX-M in Turkey. J Antibiot (Tokyo) 2013; 66: 647-50. 